\title{
Metabolic Biomarkers in Young Soccer Players during a Competitive Season
}

\author{
Rafael Guilherme Fornel ${ }^{1}$, Eike Bianchi Kohama ${ }^{1}$, Carlos Eduardo Martinelli Junior ${ }^{2}$ and Hugo Tourinho \\ Filho $^{1^{*}}$ (iD
}

${ }^{1}$ School of Physical Education and Sport of Ribeirao Preto, University of São Paulo, Brazil

${ }^{2}$ Department of Pediatrics, Ribeirao Preto Medical School, University of São Paulo, Brazil

\begin{abstract}
Purpose: The aim of this study was to verify the kinetics of the GH, IGF-I, IGFBP-3, Creatine Kinase (CK) and Lactate Dehydrogenase (LDH) at different moments in a soccer competition.

Material and Methods: The levels of GH, IGF-I and its binding protein, IGFBP3, CK, and LDH were monitored in the initial, intermediate and final phases of the competitive season ( $n=11 ; 7$ months), before and after standardized training sessions (STS).

Results: The variation in IGF-I levels after STS was higher in the intermediate compared to the final evaluation $(p=0.04)$. For the IGFBP-3 values the differences occurred (between-phases), (pre-intermediate $\times$ pre-final, $p=0.03$ ) and (postintermediate $\times$ post-final $p=0.04$ ). It was not possible to identify significant differences in CK values when comparing different moments of the competition. However, it was possible to identify a significant difference when comparing pre and post STS values (intra-phase), in the initial phase of the competition $(p=0.05)$, and in the intermediate phase $(p=$ 0.05). No significant differences were observed in GH and LDH values in any of the analyzes during the championship.

Conclusion: It was concluded that IGF-I was sensitive to the acute and chronic effects of the training, exhibiting biphasic kinetics during the championship. IGFBP-3 was sensitive to the chronic effects of training. CK was sensitive only pre and post STS in the initial and intermediate phases. The values of GH and LDH did not differ at any moment.
\end{abstract}

\section{Keywords}

GH/IGF-I axis, CK, LDH, Adolescents

\section{Introduction}

Modern soccer has undergone various transformations and in recent decades there has been an increase in the number of competitive matches over the course of a season [1]. Some teams compete in regional, national, and international competitions at the same time, and in this scenario, it is not uncommon for a team to play 2 to 3 matches in a week.

According to Scott, et al. [2], in soccer, effective monitoring strategies require the tracking of variables sensitive to physiological alterations that occur during performance in training activities and matches throughout a season, in order to increase the protection of the athlete and their maximum participation in training and competitions.

A soccer game can cause potential disturbances in the muscular, endocrine, and immune systems, thus necessitating the development of specific strategies for post-game recovery [3].

Within this line of reasoning, it is possible to verify a strong relationship between training/physical exercise and anabolic function caused by the action of the GH/IGF-I axis, a system of growth mediators, receptors, and binding proteins that control somatic and tissue growth. In this sense, baseline levels of GH and IGF-I are positively correlated with muscle mass and physical fitness in children, adolescents, and adults [4].

Researchers evaluated the effects of soccer training on young players at different times of the season and found an increase in $\mathrm{GH}$ values at the beginning of the season and sta-

*Corresponding author: Hugo Tourinho Filho, School of Physical Education and Sport of Ribeirao Preto, University of São Paulo, Avenida Bandeirantes, 3900, Monte Alegre, Ribeirão Preto, SP CEP: $14040-900$, Brazil

Accepted: July 22, 2020

Published online: July 24, 2020

Citation: Fornel RG, Kohama EB, Junior CEM, et al. (2020) Metabolic Biomarkers in Young Soccer Players during a Competitive Season. Arch Sports Med 4(2):209-214 
bilization in the middle and end of the competitive period, while the levels of IGF-I and IGFBP3 remained constant [5]. For the authors, the effects of training on the GH and IGF-I kinetics remain inconclusive.

Researchers have also investigated indicators sensitive to training status, caused by the effect of soccer practice on muscles, through monitoring of creatine kinase (CK) and lactate dehydrogenase (LDH), characterized as reliable biomarkers for verifying muscle damage [6].

The literature has indicated that there seems to be a fine line between anabolic responses and catabolic responses to exercise that determines the efficiency of the training program [7]. If the anabolic response is stronger, the training program will probably lead to improved fitness, while a greater catabolic response and, particularly, if it persists for a long time, may lead to overtraining [7].

For this reason, the development and implementation of strategies that monitor recovery after intense training or matches are therefore of interest to both professionals and coaches, as these data could support important decisions, both in the pre-season periods and during competitions.

Based on this assumption, the present study aims to investigate the kinetics of GH, IGF-I, IGFBP-3, and CK and LDH enzymes during a soccer competition in young soccer players.

\section{Material and Methods}

\section{Sample}

Initially the study was composed of 21 adolescent soccer players in the $\mathrm{U}-15$ category in a competitive season. Eleven players participated in this study, who met the following inclusion criteria: Blood collection and completing standard training sessions (STS) over the 7 months of the study.

The athletes had these values represented in mean and standard deviation of height $(174.76 \pm 7.59 \mathrm{~cm})$, body mass $(62.05 \pm 7.84 \mathrm{~kg})$ and age (14.93 \pm 0.12 -years-old). They were informed verbally and in writing, through the Informed Consent Form, of the procedures that would be adopted during the research.

The study protocol conforms to the ethical guidelines of the 1975 Declaration of Helsinki and it was approved by the Local Ethics Committee.

\section{Data collection}

After being informed verbally and in writing, through the Free and Informed Consent Term regarding the procedures that would be adopted, blood was collected from the players at three different moments during a competition period. The blood collections were performed before the start of a standardized training session (STS), preceded by 30 minutes of rest and 60 minutes after the end of the session, with an interval between pre-training collection and post-training collection of approximately $3 \mathrm{~h} 30 \mathrm{~min}$. All procedures occurred in the initial phase, intermediate phase, and final phase of the athletes' competitive season, which lasted approximately seven months.

\section{Serum determination of GH, IGF-I, IGFBP-3}

Determination of serum concentrations of GH, IGF-I, and IGFBP-3 were performed by specific immune colorimetric assays, using a commercial kit (Immulite 2000, Siemens, Los Angeles, CA) and the serum concentrations were given in $\mathrm{ng} / \mathrm{mL}$.

\section{Determination of plasma CK and LDH activity}

Plasma activities of CK and LDH were determined with commercial kits from Bioliquid ${ }^{\circ}$ (Pinhais, Brazil). Calculations of $\mathrm{CK}(\mathrm{U} / \mathrm{A})$ and $\mathrm{LDH}(\mathrm{U} / \mathrm{A})$ activities in the samples were performed using the equation $\mathrm{CK}=8252 \times \Delta$ absorbance/minute and $\mathrm{LDH}=8321 \times \Delta$ respectively.

\section{Team sport training session - soccer}

The standardized training session (STS) used for the analysis of variables throughout the season was regulated through the following steps: 30 minutes of rest followed by the first collection; warm-up using running followed by multi-jumps over an elastic rope; collective (two touches) with a small field lasting $20 \mathrm{~min} \times 20 \mathrm{~min}$ with the whole team - the collective strategy with two touches and reduced space allows the participation of all players during the training session and at an intensity similar to official games [8]; cool-down with 10 min light jogging.

\section{$24 \mathrm{~h}$ diet recall}

Multiple surveys were administered aiming to quantify the amount of food consumed in 24-hour period prior to the interview. To calculate the data obtained from multiple 24 hours dietary recalls, the DietWin ${ }^{\circledR}$ program was used, which is a support system for nutritional assessment and diet prescription. The parameters established by the American Dietetic Association and Dietary Reference Intakes (DRIs) for age and level of physical activity performed were used to analyze the data obtained.

\section{Statistical analysis}

For the analysis of the kinetics of the GH/IGF-I/IGFBP-3 system at different moments of the competitive season (initial $x$ intermediate $x$ final - between-phases) and before and after the standardized training session (pre $\times$ post- intra-phase) Friedman and Wilcoxon's non-parametric tests were used, respectively, adopting a significance level of 0.05 . The Wilcoxon test was applied in order to identify where the differences occurred.

For the analysis of plasma concentrations of CK and LDH enzymes, ANOVA was used for repeated measures at different moments of the competitive period. For comparisons between pre and post STS, the Student's " $t$ " test was used for paired samples.

\section{Results}

Regarding the IGF-I values (Table 1 ) analyzed intra-phase (pre $\times$ post STS), significant differences were observed in the results obtained in the evaluation of the intermediate phase of the competition due to an increase in IGF-I levels after STS $(459.9 \pm 67.52$ versus $519 \pm 115.39 ; p=0.05)$, while the final 
Citation: Fornel RG, Kohama EB, Junior CEM, et al. (2020) Metabolic Biomarkers in Young Soccer Players during a Competitive Season. Arch Sports Med 4(2):209-214

Table 1: Kinetics of serum concentrations of IGF-I and IGFBP-3, observed in soccer players in the U-15 category $(n=11)$ at different moments of the competitive season.

\begin{tabular}{|c|c|c|c|}
\hline \multicolumn{4}{|c|}{ Competitive Season } \\
\hline & Initial & Intermediate & Final \\
\hline \multicolumn{4}{|l|}{ IGF-I (ng/mL) } \\
\hline Pre-training & $445.54 \pm 91.1$ & $459.9 \pm 67.52^{*}$ & $460.72 \pm 94.65^{*}$ \\
\hline Post-training & $455.09 \pm 87.75$ & $519 \pm 115.39^{*}$ & $428.72 \pm 87.33^{*}$ \\
\hline$\Delta_{\text {IGF-I }}$ & $9.54 \pm 3.36$ & $59.09 \pm 95^{+}$ & $-32 \pm 49$ \\
\hline \multicolumn{4}{|c|}{ IGFBP-3 (ng/mL) } \\
\hline Pre-training & $4.46 \pm 0.63$ & $4.91 \pm 1.04^{\mathrm{a}}$ & $4.6 \pm 0.97^{a}$ \\
\hline Post-training & $4.61 \pm 0.79$ & $5.53 \pm 1.66^{b}$ & $4.5 \pm 0.79^{b}$ \\
\hline$\Delta_{\text {IGFBP-3 }}$ & $0.14 \pm 0.16$ & $0.62 \pm 0.65$ & $-0.01 \pm 0.17$ \\
\hline \multicolumn{4}{|l|}{$\mathrm{GH}(\mathrm{ng} / \mathrm{mL})$} \\
\hline Pre-training & $2.85 \pm 2.94$ & $4.29 \pm 4.48$ & $7.35 \pm 7.36$ \\
\hline Post-training & $1.75 \pm 2.31$ & $4.11 \pm 6.15$ & $2.5 \pm 2.57$ \\
\hline$\Delta \mathrm{GH}$ & $-1.1 \pm 0.63$ & $-0.18 \pm 1.67$ & $-4.85 \pm 4.79$ \\
\hline
\end{tabular}

*Difference between moments Pre and Post - STS $p \leq 0.05$

${ }^{a}$ Difference between moments intermediate - pre STS Vs. final - pre STS $p=0.03$

${ }^{b}$ Difference between moments intermediate - post STS Vs. final - post STS $p=0.04$

$\Delta=$ (post-training dosage - pre-training dosage).

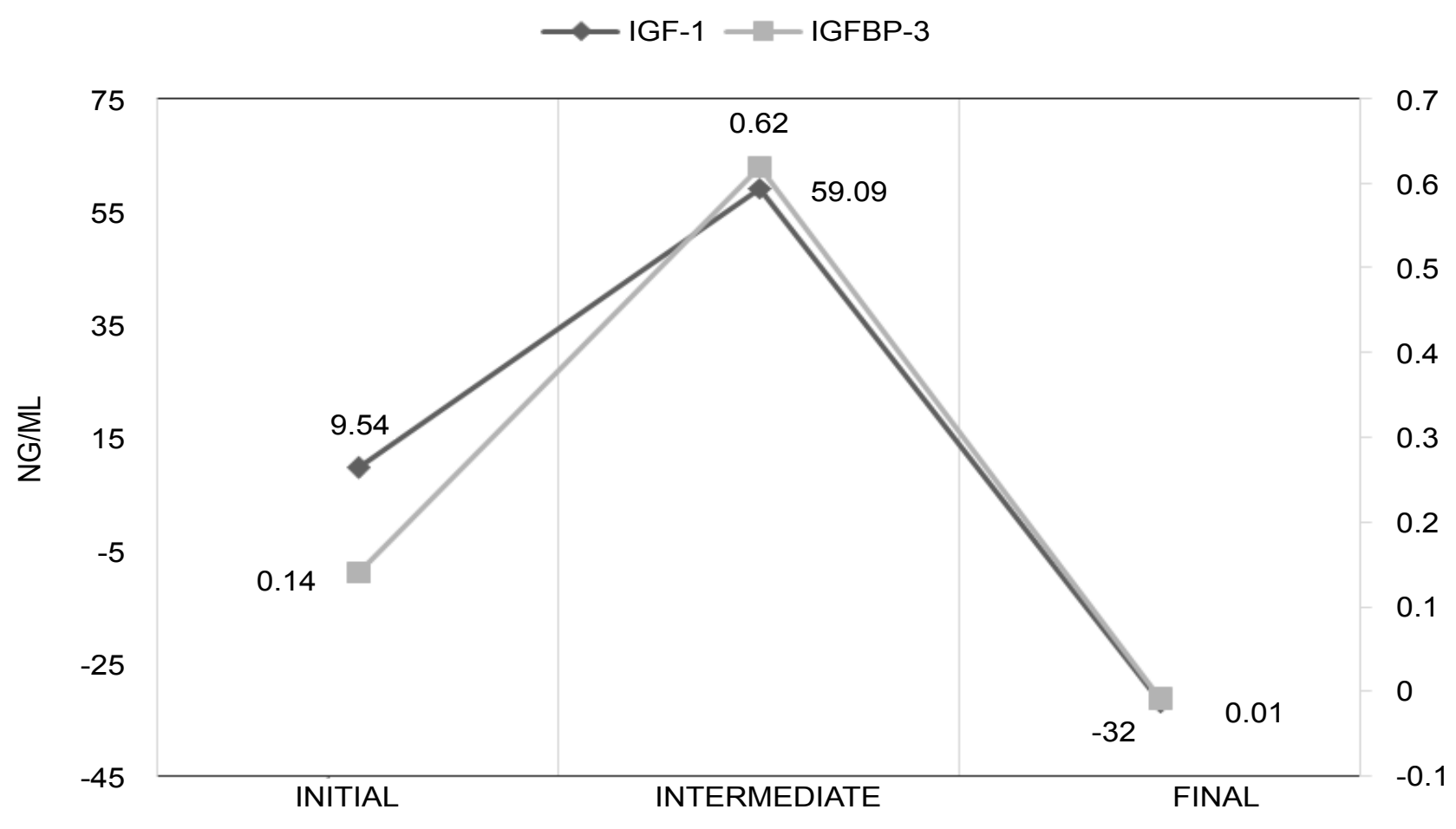

Figure 1: Kinetics of $\triangle I G F-I$ and $\triangle I G F B P-3$ at different moments of the competitive season $(n=11)$. Difference intermediate $\times$ final phase $(p=0.04)$.

stage was characterized by a reduction in IGF-I levels after STS (460.72 \pm 94.65 versus $428.72 \pm 87.33 ; p=0.04)$.

Regarding the inter-phase analysis (initial $\times$ intermediate $x$ final phase), the variation in IGF-I levels after STS was statistically greater in the intermediate phase of the competition when compared to the final phase $(59.09 \pm 95$ versus $-32 \pm$
49; $p=0.04)$, evidencing a catabolic effect at the end of the competition (Table 1).

For the IGFBP-3 values, the differences occurred during the competition at two moments (intermediate; $4.9 \pm 1.0$ versus $4.6 \pm 1.0 ; p=0.03$; and final; $5.5 \pm 1.7$ versus $4.5 \pm 0.8$; $p=0.04)$. For the intra-phase comparisons (pre $\times$ post), no 
statistically significant differences were observed for IGFBP-3 values. Regarding the $\mathrm{GH}$ values, no alterations, either acute or chronic, were identified (Figure 1).

It was also not possible to identify significant differences in CK values when comparing the three different moments of the competition (initial $x$ intermediate $\times$ final). However, it was possible to identify a significant difference when comparing the pre and post STS values (intra-phase), specifically in the initial phase of the competition $(188.6 \pm 86.78$ versus $267.02 \pm 87.5 ; p=0.05)$, as well as in the intermediate phase (194.15 \pm 107.46 versus $363.09 \pm 226.2 ; p=0.05)$.

Regarding the LDH values measured, no acute or chronic alterations were identified and the values remained constant, regardless of the moment of competition (Table 2 ).

\section{Discussion}

The main finding of the present study was to verify, for the first time in this sport modality, that the IGF-I is sensitive to the acute and chronic effects of training, presenting alterations in its kinetics in two phases throughout the competition, corroborating the hypothesis of kinetics in the literature by a series of authors, as the "Theory of the two phases" $[7,9,10]$.

It has been suggested that the GH/IGF-I axis exhibits biphasic behaviour during the training season ("Theory of the two phases") $[7,9,10]$; with a catabolic phase characterized by a reduction in hormone concentrations lasting 3 to 5 weeks followed by an anabolic phase that takes place after 5 to 6 weeks of training, characterized by an increase in hormone concentrations $[7,9]$. The increase in IGF-I concentrations observed following a long training program (4 to 9 weeks) has been supported by studies using animal models, which reported increases in IGF-I gene expression in the muscular-skeletal tissue and in circulating IGF-I concentrations [7,9]. However, it remains unclear whether the biphasic behaviour of the $\mathrm{GH}$ / IGF-I axis also applies to humans during a prolonged training program [9].

According to Eliakim and Nemet [7] the very fine balance between the anabolic and in inflammatory/catabolic response to exercise will determine the effectiveness of exer- cise training and the health consequences of exercise. If the anabolic response is stronger than the catabolic response, exercise will probably lead ultimately to increased muscle mass and improved fitness. A dominant catabolic response, especially if it persists for long duration, may lead to overtraining. The response of these mediators to different types of sports, training sessions, or training protocols can be used as an objective tool to monitor the training load and to better plan training cycles throughout the competitive season [9].

Although $\mathrm{GH}$ concentrations do not differ significantly during the season, IGF-I result changes may have indicated an "anabolic" phase evidenced by an increase in the IGF-I concentrations in the intermediate phase of the competition when compared to the final phase. The IGFBP-3 was shown to be sensitive to the chronic effects of training, although it was not possible to identify differentiated kinetics when comparing before and after a standard training session.

Mejri, et al. [5] studied the effects of a soccer training program on GH and IGF-I levels in 13 young soccer players using a submaximal test performed on a cycle ergometer. The authors found that GH levels increased with exercise performed during the submaximal test, but significantly higher responses were observed at the beginning of the season when compared to the middle and end of the training program. Regarding the IGF-I levels, these remained stable throughout the season, and it was not possible to observe the hypothesis about the possibility of the GH/IGF-I axis kinetics occurring in two phases: Catabolic and anabolic throughout a training season [5].

It is possible the differences in results found in the present study with those verified in the study conducted by Mejri, et al. [5] can be due to the different experimental designs used. While the present study sought to use a methodology closer to the reality of the sport (real life), the study by Mejri, et al. [5] used tests that deviate from the specificity of the modality.

The causes of the decrease in IGF-I levels during periods of intense training or what represents an adequate elevation in this substance during the finishing and reduced training phases remain controversial. However, it is believed that the

Table 2: Effects of the standardized training session on the plasma activity of CK and LDH, observed in soccer players in the U-15 category ( $\mathrm{n}$ $=11$ ) at different moments of the competitive season.

\begin{tabular}{|c|c|c|c|}
\hline \multicolumn{4}{|c|}{ Competitive Season } \\
\hline & Initial & Intermediate & Final \\
\hline \multicolumn{4}{|l|}{ CK (U/A) } \\
\hline Pre-training & $188.6 \pm 86.78$ & $194.15 \pm 107.46$ & $299.5 \pm 143.38$ \\
\hline Post-training* & $267.02 \pm 87 . .^{*}$ & $363.09 \pm 226.2^{*}$ & $324.3 \pm 181.45$ \\
\hline$\Delta$ of alteration & 78.41 & 168.94 & 25.24 \\
\hline \multicolumn{4}{|l|}{ LDH (U/A) } \\
\hline Pre-training & $137.67 \pm 70.34$ & $193.65 \pm 129.76$ & $206.51 \pm 53.51$ \\
\hline Post-training & $178.52 \pm 129.83$ & $209.53 \pm 52.33$ & $238.28 \pm 75.28$ \\
\hline$\Delta$ of alteration & 40.84 & 15.88 & 31.77 \\
\hline
\end{tabular}

${ }^{*}$ Significant difference in relation to Pre and Post STS ( $\left.\leq \leq 0.05\right)$. 
inability to increase circulating IGF-I levels before an important competition should be understood as a warning sign for athletes and coaches, as it may indicate that the athlete has not yet reached their optimal athletic state [10].

Regarding the effects of the standardized training session (STS) on the plasma activity of CK and LDH, observed in soccer players in the U-15 category at different moments of the competitive period, it was possible to identify significant differences only for the CK values before and after STS in the results obtained in the initial phase of the competition, and also in the intermediate phase.

Physiological understanding of the reasons for the increase in CK after exercise is not yet fully elucidated, as it consists of a modality with an intermittent characteristic, involving activities such as jumps, accelerations, decelerations, and changes of directions [11]. The damage that occurred may be related to the amount of eccentric activation of the muscle. It has been shown that the lower muscle activity found in this type of muscle action, when compared to the concentric action, can induce greater mechanical stress on the active muscle fibers, increasing the incidence of micro traumas $[12,13]$.

Data similar to the present study regarding the difference in CK pre- and post- STS in the initial and middle stages of competition were found in the study conducted by Aquino, et al. [14]. The authors studied the alterations in CK and LDH during a simulated soccer game in which two blood samples were taken, one pre and another 30 min post-game. Through the analysis of the data it was possible to verify a strong association between the percentage of the $\mathrm{CK}$ and LDH alteration delta with the displacement patterns in the simulated game.

Observing the effects of 22 weeks of periodized training with an emphasis on technical-tactical ability on indirect indicators of CK and LDH muscle damage in 15 young soccer players (age $15.4 \pm 0.2$ years), Aquino, et al. [15] also observed a significant reduction $(p \leq 0.05)$ in plasma CK and LDH activity during the periodization.

The changes in the levels of CK can also be explained by the direct confrontation between the opponents in dispute for space or for the ball, generating a high number of physical contacts during the matches, thus being able to increase the incidence of micro traumas. Data from other collective sports modalities similar to soccer, where there are a large number of contacts and impacts with the opponent (American football and rugby), found that results with physical variables, such as tackles and impacts, are more clearly associated with increased levels of CK than the movement patterns that are common in team sports $[13,16]$.

Scott, et al. [2] followed 15 elite soccer players in a competitive English Premier League (EPL) season to determine the relationship between creatine kinase (CK) in the blood $48 \mathrm{~h}$ after a competitive match and physical performance. No significant relationship was observed between any physical performance indicator, and CK concentration measured 48 hours after the match. The authors suggest that CK concentrations are high as a result of a football match. For the authors, this evidence suggests that the CK values obtained after the exercise may not accurately reflect the changes in the activity profiles performed by soccer players during matches and training.

On the other hand, the use of the IGF-IGFBPs system as a biomarker of training status is supported by a review of Nindl and Pierce [17] in which there is scientific evidence that IGF-I is an important metabolic biomarker of training status in different sports.

Specifically, in the present study, the kinetics of serum concentrations of growth mediators proved to be sensitive to both acute and chronic effects of exercise, in the case of IGF-I, and chronic effects throughout competition, as observed for the IGFBP-3.

For this reason, it seems reasonable to suggest that these components of the GH/IGF-I axis may serve as important markers of training status for adolescent soccer players throughout a competitive season.

Others factors can also influence the GH/IGF-I axis beyond exercise, such as the reduction of pre-competition weight, high carbohydrate and lipids diet [18]. The analysis of the dietary recall performed with adolescent soccer players allows excluding the influence of the diet in our results as the diet recall was similar in the 3 moments of evaluation.

\section{Conclusions}

- IGF-I is sensitive to the acute and chronic effects of training, with alterations in its kinetics in two phases throughout the competition;

- IGFBP-3 was shown to be sensitive to the chronic effects of training, and it is not possible to identify differentiated kinetics when comparing before and after a standard training session;

- The GH did not present any acute or chronic pattern of alterations throughout the championship;

- CK was shown to be sensitive only to the acute effects of training, it being possible to identify differentiated plasma activity when comparing before and after a standard training session, however this change was identified only in the initial and intermediate stages of the championship;

- LDH plasma activity remained constant throughout the season, demonstrating that it was unaffected by the acute or chronic effects of soccer training in adolescent athletes.

It is believed that it is important to carry out further field studies that can accompany training programs for young athletes and their possible effects on the body, including measures of cytokine, insulin, and glycemic levels, as well as other IGF-I binding proteins (IGFBP-1) that may explain the decrease in growth mediators at certain stages and advance the possible repercussions of this suppression on the growth and development of adolescent athletes.

\section{Disclosure Statement}

The author does not have any financial interest and did not receive any financial benefit from this research. 


\section{Conflict of Interest}

The author states no conflict of interest.

\section{Author Contributions}

All the authors have accepted responsibility for the entire content of this submitted manuscript and approved submission.

\section{References}

1. Thorpe R, Corolinem S (2012) Muscle damage, endocrine, and immune marker response to a soccer match. J Strength Cond Res 26: $2783-2790$.

2. Scott A, Malone JJ, Morgans R, et al. (2016) The relationship between physical match performance and $48 \mathrm{~h}$ post-game creatine kinase concentrations in English Premier League soccer players. Int J Sports Sci Coach 11: 846-852.

3. Reilly T, Ekblom B (2005) The use of recovery methods post-exercise. Journal of Sports Science 23: 619-627.

4. Martinelli Junior CE, Custódio RJ, Oliveira MHA (2008) Physiology of the GH-IGF axis. Arq Bras Endocrinol Metabol 52: 717-725.

5. Mejri S, Bchir F, Ben Rayana MC, et al. (2005) Effect of training on GH and IGF-1 response to a submaximal exercise in football players. Eur J Appl Physiol 95: 496-503.

6. Ascensao A, Rebelo A, Oliveira E, et al. (2008) Biochemical impact of a soccer match - analysis of oxidative stress and muscle damage markers throughout recovery. Clin Biochem 41: 841851.

7. Eliakim A, Nemet $D(2010)$ Exercise training, physical fitness and the growth hormone-Insulin-Like growth factor-1 axis and cytokine balance. Med Sport Sci 55: 128-140.

8. Coelho DB, Rodrigues VM, Condessa LA, et al. (2008) Intensity of training sessions and official football matches. Rev Bras Educ Fis Esp 22: 211-218.

9. Eliakin A, Nemet D (2013) Exercise and the GH/IGF-1 axis. In:
Constantini N, Hackney AC, Endocrinology of Physical activity and sport. (edn), Springer Science + Business Media, New York, 69-83.

10. Tourinho Filho H, Pires M, Puggina EF, et al. (2017) Serum IGF-I, IGFBP-3 and ALS concentrations and physical performance in young swimmers during a training season. Growth Horm IGF Res 32: $49-54$.

11. Lazarim LF, Antunes-Neto JM, Da Silva FO, et al. (2009) The upper values of plasma creatine kinase of professional soccer players during the Brazilian national championship. J Sci Med Sport 12: $85-90$.

12. Tesch PA, Dudley GA, Duvoisin MR, et al. (1990) Force and EMG signal patterns during repeated bouts of concentric or eccentric muscle actions. Acta Physiol Scand 138: 263-271.

13. Johnston DR, Tim JG, Anthony JS, et al. (2014) Influence of physical contact on neuromuscular fatigue and markers of muscle damage following small-sided games. J Sci Med Sport 17: 535540.

14. Aquino RL, Gonçalves LG, Vieira LH, et al. (2016) Biochemical, physical and tactical analysis of a simulated game in young soccer players. J Sports Med Phys Fitness 56: 1554-1561.

15. Aquino RL, Goncalves LG, Vieira LH, et al. (2016) Periodization training focused on technical-tactical ability in young soccer players positively affects biochemical markers and game performance. J Strength Cond Res 30: 2723-2732.

16. Kraemer WJ, Spiering BA, Volek JS, et al. (2009) Recovery from a national collegiate athletic association division I football game: Muscle damage and hormonal status. J Strength Cond Res 23: 2-10.

17. Nindl BC, Pierce JR (2010) Insulin-like growth factor I as a biomarker of health, fitness, and training status. Med Sci Sports Exerc 42: 30-49.

18. Galassetti P, Larson J, Iwanga K, et al. (2006) Effect of a high-fat meal on the growth hormone response to exercise in children. $J$ Pediatr Endocrinol Metabol 19: 777-786.

DOI: $10.36959 / 987 / 254$

Copyright: (C) 2020 Fornel RG, et al. This is an open-access article distributed under the terms of the Creative Commons Attribution License, which permits unrestricted use, distribution, and reproduction in any medium, provided the original author and source are credited. 